\title{
Using dynamic diversity to achieve durable disease resistance in agricultural ecosystems
}

\author{
Bruce A. McDonald \\ Plant Pathology Group, Institute of Integrative Biology, ETH Zurich, 8092 Zurich, Switzerland \\ E-mail: bruce.mcdonald@usys.ethz.ch
}

Genetically resistant cultivars form the foundation of disease management for most crops. Populations of resistant cultivars grown at the farm and landscape scales keep many important fungal, bacterial and viral diseases in check, sometimes for many years. But eventually, most resistant cultivars fail and an epidemic ensues, often causing significant losses in crop yield and quality. In this letter, disease resistance is considered as a population phenomenon, exhibited mainly at the scale of fields, farms and landscapes and encompassing millions or billions of plants, rather than at the level of individual plants or specific plant tissues, even though cultivar resistance is often measured based on symptoms of individual plants or tissues. But it should be noted that the failure of resistance at the population or landscape level generally begins with a failure of resistance at the individual level.

Disease resistance fails because pathogens evolve. Evolution requires genetic diversity. Genetic diversity is affected by mutation, population size, recombination, gene flow, and selection, the same factors that affect an organism's population genetics. Thus the keys to developing strategies for breeding durable disease resistance lie in understanding pathogen population genetics.

The root of the problem of pathogen evolution is the lack of diversity in agricultural ecosystems (agroecosystems). Since the invention of agroecosystems $\sim 12,000$ years ago, crop genetic diversity has declined steadily in agroecosystems globally to facilitate gradual improvements in agricultural production systems, including innovations such as tillage, fertilization, controlled irrigation, and mechanization. The decline in crop genetic diversity at the field scale accelerated rapidly during the last 100 years to increase the efficiency of food production and feed the burgeoning human population. The large-scale mechanization of planting and harvesting operations and the green revolutions that swept through agriculture led to replacement of locally-adapted but genetically diverse land races by genetically uniform but broadly-adapted, highyielding, dwarf cultivars, further depleting genetic diversity and increasing the environmental homogeneity present in agroecosystems worldwide. The highly mechanized modern industrial agroecosystems now found around the world are extremely productive food factories, but also are highly effective incubators of pathogen evolution (as described in Stukenbrock \& McDonald, 2008). As a result of the increased planting density and genetic uniformity of host populations in agroecosystems compared to natural ecosystems, pathogen transmission became easier, enabling an increase in pathogen virulence (Read, 1994). The same factors increased pathogen population sizes, which led to more genetic diversity for selection to act upon by increasing the total number of mutations available at the field scale while simultaneously lowering the effects of genetic drift. Due to these changes in agroecosystems over time, pathogen evolutionary potential likely increased as agricultural pathogens (including fungi, bacteria and viruses) became domesticated and adapted to the agroecosystem environment.

Other factors unique to agroecosystems are also likely to contribute to more rapid pathogen evolution. The higher host density found in agroecosystems compared to natural ecosystems allows agricultural pathogens to exist at a higher density. This higher pathogen density increases the likelihood of multi-infections in the same plant by different genotypes of the same pathogen (e.g. Linde et al. 2002; Keller et al. 1997; McDonald et al. 1999). Multi-infections have long been thought to favor the development of higher virulence (defined here as the amount of damage done to the plant, but often referred to as aggressiveness or quantitative virulence in plant pathology) as a side effect of competition among strains for host resources (Van Baalen \& Sabelis, 1995). The higher host and pathogen density found in agroecosystems also increases the likelihood of co-infection by different pathogen species, which also is thought to select for higher virulence as a result of competition among pathogen species for the same host resources. In addition, co-infection by different pathogen species increases the likelihood of horizontal transfer of genes among pathogens infecting the same host, as reported recently for several wheat pathogens (e.g. Friesen et al. 2006; Gardiner et al. 2012; McDonald et al. 2013).

Modern agroecosystems are very well structured to produce high quality produce that can be eaten directly (e.g. rice or apples) or processed into higher value goods 
(e.g. wheat into bread, maize into corn chips or barley into beer). The increasing genetic uniformity of the major crops facilitated the large-scale mechanization of agriculture and simplified all aspects of food production, while increasing crop yield and quality, improving food processing properties, keeping food prices low and liberating workers for other industries and activities (e.g. only $2 \%$ of the USA population is farmers, yet enough surplus food is generated to enable significant grain exports). The basic trade-off in all agroecosystems is that the increased environmental and genetic uniformity that enables more efficient food production also facilitates pathogen evolution and increases the risk of significant losses due to disease. Thus we are confronted with a dilemma: How can we maximize the many benefits of modern industrial agriculture while minimizing the risks of disease epidemics in these agroecosystems? Is it possible to achieve long-lasting and stable (i.e. durable) disease control, especially if it is based largely on the resistance genes bred into resistant cultivars?

Truly durable resistance (i.e. highly effective resistance that prevents a damaging epidemic over temporal scales measured in decades or centuries and spatial scales measured in tens of thousands or millions of hectares) will not be achieved unless we increase the overall diversity present in agroecosystems at both the farm and landscape spatial scales. The diversity will need to be dynamic, changing regularly over both time and space, to significantly slow the rate of pathogen adaptation. How can we increase total agroecosystem diversity at the farm and landscape scale without losing the advantages that come with increasing crop uniformity? Fortunately, a wide array of low-technology, medium-technology and high-technology strategies can be used to increase both spatial and temporal diversity in agroecosystems. Many of these strategies are well known and oriented around crop husbandry, requiring no additional breeding effort. These crop husbandry strategies include improved crop rotations, species intercropping, planting smaller fields to increase crop heterogeneity at the farm and landscape scales, introducing new crop species into existing agroecosystems, and increasing the overall genetic diversity present in existing crop species by introducing new genes from their wild relatives. I will focus the rest of this letter on plant breeding strategies.

Breeding strategies for durable resistance can include both major gene resistance (MGR) and quantitative resistance $(\mathrm{QR})$ used alone or in combination (MGR and QR are defined in McDonald \& Linde 2002a). But to be successful over the long-term, any breeding program will need to embrace the concept of dynamic turnover to insure the durability of its resistant products, be they newly identified resistance genes or resistant cultivars. Dynamic turnover means that the resistance genes and resistant cultivars will need to change at regular intervals over time and space. Static, long-term use of any form of resistance will impose strong directional selection on the corresponding pathogen population that will favor evolved pathogen strains that can overcome the resistance, leading to an eventual epidemic. The "bust" of MGR used singly or in pyramids has been widely documented for many decades in all major crops (e.g. McDonald \& Linde, 2002a). QR used alone is also likely to erode over time as a result of pathogen evolution, though the erosion may be more difficult to document (see appendix in McDonald \& Linde 2002a). It is likely that pyramids (stacks) of QR and MGR (e.g. Brun et al. 2010) will also fail after prolonged use, but dynamic turnover of MGR and QR pyramids containing unique combinations of resistance genes will be more likely to provide durable resistance. Advanced genetic technologies will increasingly provide new cultivars with many different forms of resistance created by natural processes or creative minds (e.g. Koch et al. 2013), but even the most novel humanengineered resistance is likely to fail if it is deployed in a static monoculture. Dynamic turnover of resistant cultivars and resistance genes will be an important aspect of any strategy that leads to durable resistance.

In general, breeding strategies for durable resistance can focus on increasing crop gene diversity or increasing crop genotype diversity to slow pathogen evolution. The most successful resistance breeding programs will likely incorporate both gene and genotype diversity into their breeding strategy.

Genotype-based approaches are oriented around breeding strategies that lead to a variable host population composed of many different genotypes at the field scale. Examples of breeding outcomes that utilize genotype diversity include composite crosses (synthetic landraces), cultivar mixtures, and cisgenic or transgenic multilines. Over the last 20 years, we conducted three different field experiments in three locations and with three different cereal pathogens that showed that 2-component cultivar mixtures can significantly slow pathogen evolution (Abang et al. 2006; Sommerhalder et al. 2011; Zhan et al. 2002; Zhan \& McDonald 2013a, b). The consistent findings in these experiments increase our confidence that breeding approaches that increase genotype diversity within fields will provide a significant step forward on the path towards durable resistance.

Gene-based approaches are oriented around breeding strategies that lead to a genetically uniform host population at the field scale. Examples of breeding outcomes that utilize gene diversity include MGR or QR gene rotations based on isogenic lines (e.g. the components of a multiline can be grown in a temporal rotation instead of in a mixture), MGR or QR pyramids (stacks) built into a single cultivar, and targeted combinations of MGR and QR achieved using marker-assisted selection (MAS, e.g. Brun et al. 2010) or the resistance gene (R-gene) cassette.

The R-gene cassette is a concept for an advanced breeding strategy based on genetic engineering that has not yet been created, but is a logical target for biotechnology (Wulff et al. 2011) that could probably be achieved during the next decade. The R-gene cassette is a cluster of different 
R-genes that have been spliced together in the lab using genetic engineering technologies, with the different R-genes encoding combinations of QR (e.g. Lr34, Krattinger et al. 2009) and/or MGR that work against a broad array of pathogens or pathogen strains (Figure 1). The engineered cassette carries appropriate flanking sequences and selectable markers to allow stable integration into a conserved location in the plant genome that is already occupied by a naturally evolved R-gene cluster (Michelmore \& Meyer, 1998). New R-gene cassettes carrying different combinations of resistance genes can be generated in the lab and used to replace existing R-gene cassettes through homologous recombination as needed to ensure dynamic turnover of resistance genes and in response to the emergence of new, virulent pathotypes. By using the same integration and gene expression vector, different R-gene cassettes can be deployed in the same crop growing in different geographical locations to reflect differences in agroecosystems and corresponding differences in the local disease complexes. Plants evolved natural R-gene clusters as a strategy for defense against pathogens over millions of years of coevolution with their pathogens. The R-gene cassette is thus based on imitating natural evolutionary processes, but with humans controlling the process by putting together combinations of resistance genes that control the pathogens that are most relevant in each agroecosystem.

A final consideration in deciding on an appropriate breeding strategy is the nature of the crop and the amount of capital (human, monetary or institutional) available to invest in durable resistance breeding strategies. Subsistence farmers growing rice in 0.5 ha rice paddies in Laos are likely to benefit from a different breeding strategy than industrial farmers growing rice in 1000 ha rice fields in Louisiana.
Similarly, an apple orchard or oil palm plantation planted to a long-lived clone that is expected to produce fruit over a period of 15-50 years will likely need a different breeding strategy than an arable farm that cultivates annual cereals, where new wheat or maize cultivars can be introduced every few years. With these ideas in mind, I prepared two new decision diagrams (Figure 2) to complement the ones shown earlier (McDonald \& Linde 2002a, 2002b) to illustrate which strategies may be most appropriate in different agroecosystem settings.

The new decision diagrams take into account the type of crop, contrasting crops with a rapid cultivar turnover (e.g. annual cereals) with crops that show a low cultivar turnover (e.g. perennial trees and vines such as oil seed palms, apples and grapes). They also consider the overall scale and type of farming, ranging from well-capitalized, highly mechanized, large-scale industrial farms to less mechanized, less-capitalized and smaller-scale subsistence or family farms. The diagrams also consider the type of technology that is likely to be available to the farmer, which generally reflects the overall amount of capital investment needed to implement and sustain the breeding effort. For example, low-technology options (requiring little capital investment) that can increase diversity at the farm scale include deployment of traditional cultivars carrying single MGR or QR, gene/cultivar rotations through time and space, synthesis of composite crosses (modern landraces) and use of cultivar mixtures. High-technology options (requiring significant capital investment) that can increase diversity at the farm scale include cisgenic or transgenic multilines containing QR or MGR created using genetic engineering (GE) technologies, pyramids of QR and MGR created using marker-assisted selection (MAS), and creation of R-gene

\begin{tabular}{|c|c|c|c|c|c|c|c|c|c|}
\hline MGR1 & MGR2 & QR1 & MGR1 & MGR2 & QR1 & MGR1 & MGR2 & QR1 & QR1 \\
\hline MGR3 & MGR4 & QR2 & MGR3 & MGR4 & QR2 & MGR3 & MGR4 & QR2 & MGR1 \\
\hline MGRS & MGR6 & QR1 & MGR1 & MGR2 & QR1 & MGR1 & MGR2 & QR1 & QR1 \\
\hline MGR7 & MGR8 & QR2 & MGR3 & MGR4 & QR2 & MGR3 & MGR4 & QR2 & MGR1 \\
\hline
\end{tabular}

FIGURE 1 - Conceptual organization of an R-gene cassette carrying a cluster of 10 R-genes. An R-gene cassette carries genes that encode major gene resistance (MGR) and quantitative resistance (QR) to the most common pathotypes of the most common diseases located in a region. Each cassette carries conserved selectable markers and flanking sequences that enable targeted integration into an existing R-gene cluster that is conserved within a crop species (e.g. one cassette backbone vector would be used for wheat and a different vector would be used for soybeans). Several cassettes carrying different combinations of R-genes would be prepared for each region to enable a dynamic turnover of the R-gene cassette. Different R-gene cassettes can also be deployed spatially in mixtures or temporally in rotations. Each colored rectangle represents a different R-gene encoding either MGR or QR. Different genes or alleles for each MGR or QR are given different numbers, signifying that they recognize different pathogen effectors (MGR) or have different mechanisms of QR. Colors represent R-genes effective against different diseases. R-gene cassettes A and B can be alternated in a region where diseases represented by orange, blue, green and magenta are common. R-gene cassettes $\mathrm{C}$ and $\mathrm{D}$ can be alternated in a region where diseases represented by orange, red, gray and light orange are common. As local pathogen populations adapt to particular R-genes in each cassette, old cassettes can be modified and new cassettes can be released while the background plant genotype can remain constant, providing a high degree of crop uniformity. 
Decision Diagram A: Rapid Cultivar Turnover (annual grains)

High capital/research

investment needed

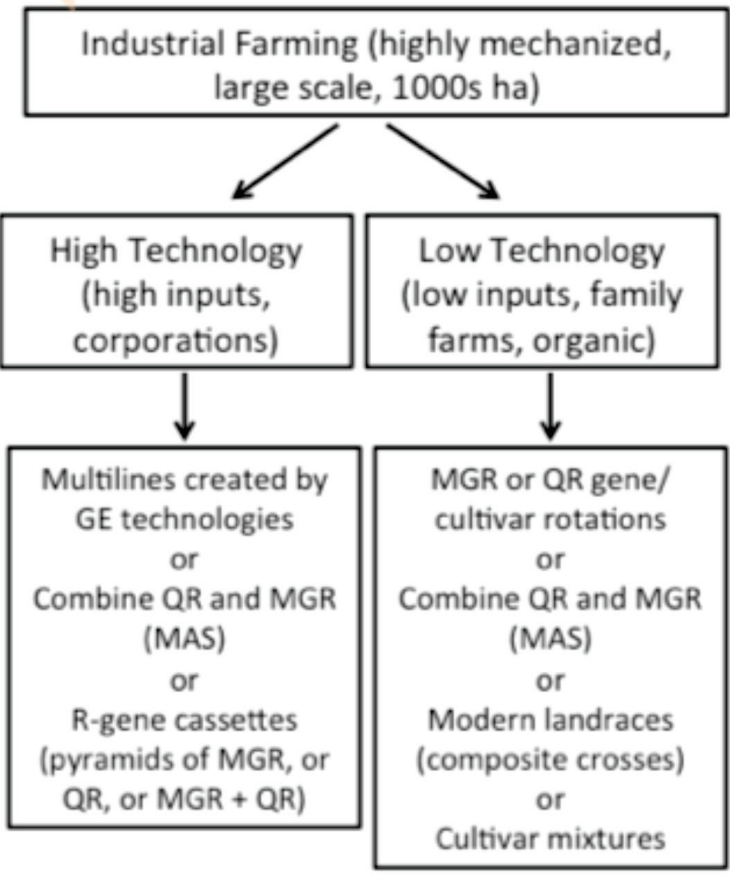

Low capital/research

investment needed

Family Farming (little mechanization, small scale, 1-100 ha)

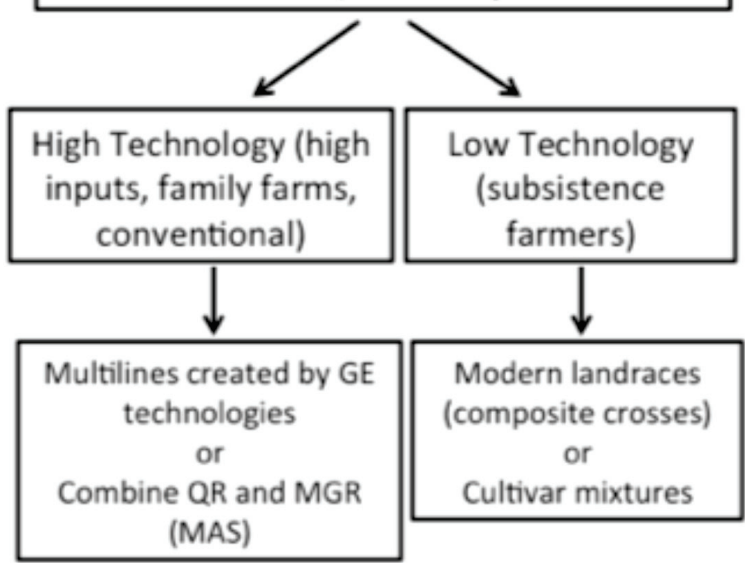

Decision Diagram B: Slow Cultivar Turnover (fruit trees, nuts)

\section{High capital/research investment needed}

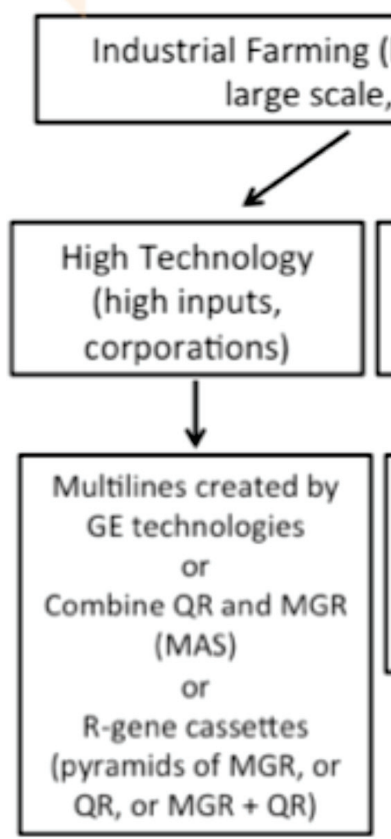

Low capital/research

investment needed

Family Farming (little mechanization, small scale, 1-100 ha)

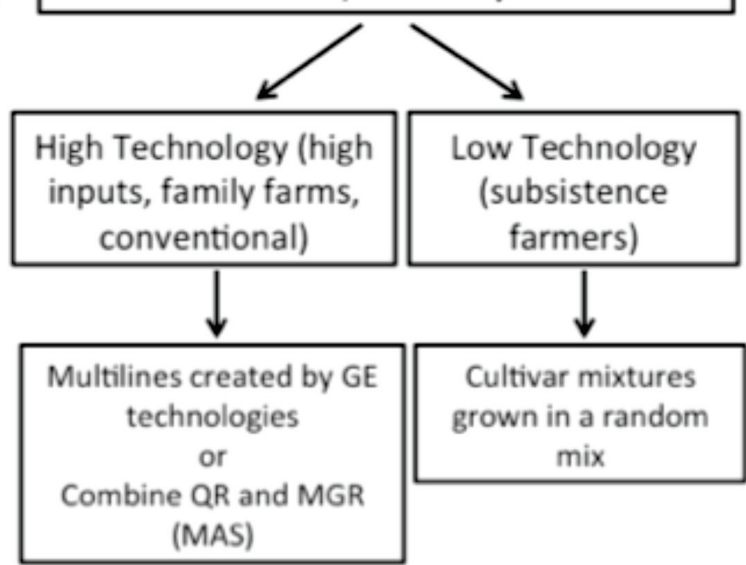

FIGURE 2 - A. Decision diagram showing breeding options for annual crops that experience a rapid cultivar turnover, such as wheat, barley and soybeans; B. Decision diagram showing breeding options for plantation and orchard crops that are long-lived and experience a slow cultivar turnover, such as oil palms, bananas, apples, grapes and nut trees. 
cassettes (pyramids of MGR, or QR, or MGR + QR) using GE technologies. It would not make sense to invest in hightechnology, capital-intensive approaches to create durable resistance to pathogens that have low evolutionary potential or for crops grown on a small scale that turnover quickly. But high value, clonal crops grown on industrial scales and confronted by rapidly evolving pathogens (e.g. black sigatoka on bananas) would be very likely to benefit from using the high technology approaches discussed here. The intention of these diagrams is to guide the breeder or plant pathologist or breeding institution to choose the strategy that is most appropriate to the agroecosystem and end-user that is the target of the breeding program.

Figure 2A considers annual crops such as wheat, rice, soybeans and maize that can experience relatively rapid cultivar turnover. Poor subsistence farmers growing annual crops in small fields are likely to save seeds from each harvest to plant future crops and are unlikely to afford high-technology crops created using GE, such as herbicideresistant crops that require additional inputs. But these farmers can readily maintain diversity in their fields by planting composite crosses synthesized by making pairwise crosses among elite germplasm (these composite crosses could be generated with assistance from NGOs or CGIAR programs) or cultivar mixtures that turnover slowly. Small scale ( 10-100 ha) lightly mechanized farms are more likely to be able to afford to buy new seed and could benefit from more advanced breeding strategies such as multilines created by GE technologies or advanced cultivars that have combined QR and MGR through MAS. Large scale and highly mechanized farms that cater to the high value organic food market and want to avoid GE technologies could increase genotype diversity by growing cultivar mixtures or composite crosses and/or increase gene diversity by implementing regular R-gene or cultivar rotations through time and space. The largest scale industrial farms that have access to the most capital and that can produce the highly uniform product desired by the food processing industries will benefit the most from GE technologies. These industrial farms can increase genotype diversity by growing isogenic multilines carrying different R-genes or increase gene diversity by growing advanced cultivars that have combined QR and MGR or cultivars carrying different R-gene cassettes.

Figure 2B considers plantation and orchard crops such as oil palms, bananas, apples, grapes and nut trees that are long-lived and likely to experience a slower cultivar turnover. Many of these crops are heavily treated with pesticides to control diseases and durable genetic resistance could significantly reduce the need for these pesticides. Subsistence farmers could maintain diversity by growing several different cultivars in a spatially random mix, while highly mechanized plantations and orchards could benefit by growing different cultivars in row mixtures (e.g. Didelot et al. 2007). Multilines created by GE technologies could provide many advantages for both family and industrial- scale farming because individual multiline components can be replaced when new pathotypes emerge that are specifically adapted to a particular R-gene. R-gene cassettes could be especially useful for industrial farming of clonal perennial crops that grow year round, such as oil palm or bananas, with new R-gene cassettes introduced regularly into a defined fraction (e.g. 10\%) of the plantation each year.

The key to successful durable resistance breeding will be to increase dynamic diversity within the agroecosystem at the field, farm and landscape scale. Static diversity (e.g. based on deployment of single MGR, single QR, or a constant MGR pyramid) is likely to fail given sufficient time as a result of continuous directional selection applied to the pathogen population. Plant breeders can increase genotype diversity by breeding for populations composed of many genotypes (e.g. composite crosses or cultivar mixtures) or they can increase gene diversity by selecting for populations composed of many genes (e.g. R-gene pyramids, GE multilines or R-gene cassettes). Many different breeding strategies should be considered that take into account pathogen population genetics as well as the specific agroecosystem, available technologies and the scales of production.

\section{REFERENCES}

Abang M, Baum M, Grando S, Ceccarelli S, Linde CC, Yahyaoui A, Zhan J, McDonald BA (2006) Differential selection on Rhynchosporium secalis during parasitic and saprophytic phases in the barley scald disease cycle. Phytopathology 96:1214-22.

Brun H, Chèvre AM, Fitt BD, Powers S, Besnard AL, Ermel M, ... \& Andrivon D (2010) Quantitative resistance increases the durability of qualitative resistance to Leptosphaeria maculans in Brassica napus. New Phytologist 185:285-299.

Didelot F, Brun L, Parisi L (2007) Effects of cultivar mixtures on scab control in apple orchards. Plant Pathology 56:1014-1022.

Friesen TL, Stukenbrock EH, Liu Z, Meinhardt S, Ling H, Faris JD, Rasmussen JB, Solomon PS, McDonald BA, Oliver RP (2006) Emergence of a new disease as a result of interspecific virulence gene transfer. Nature Genetics 38: 953-956.

Gardiner DM, McDonald MC, Covarelli L, Solomon P, Rusu A, Marshal M, Kazan K, Chakraborty S, McDonald BA, Manners JM (2012) Comparative pathogenomics reveals horizontally acquired novel virulence genes in fungi infecting cereal hosts. PLoS Pathogens 8: e1002952. doi:10.1371/journal.ppat.1002952.

Keller SM, McDermott JM, Pettway RE, Wolfe MS, McDonald BA (1997) Gene flow and sexual reproduction in the wheat glume blotch pathogen Phaeosphaeria nodorum (anamorph Stagonospora nodorum). Phytopathology 87:353-358.

Koch A, Kumar N, Weber L, Keller H, Imani J, Kogel KH (2013) Host-induced gene silencing of cytochrome P450 lanosterol C14 $\alpha$-demethylase-encoding genes confers strong resistance to Fusarium species. Proceedings of the National Academy of Sciences USA 110:19324-19329.

Krattinger SG, Lagudah ES, Spielmeyer W, Singh RP, Huerta- 
Espino J, McFadden H, Bossolini E, Selter LL, Keller B (2009) A putative $\mathrm{ABC}$ transporter confers durable resistance to multiple fungal pathogens in wheat. Science 323:1360-1363.

Linde C, Zhan J, McDonald BA (2002) Population structure of Mycosphaerella graminicola: from lesions to continents. Phytopathology 92:946-955.

McDonald BA, Linde C (2002a) Pathogen population genetics, evolutionary potential, and durable resistance. Annual Review of Phytopathology 40:349-79.

McDonald BA, Linde C (2002b) The population genetics of plant pathogens and breeding strategies for durable resistance. Euphytica 124:163-180.

McDonald BA, Zhan J, Burdon JJ (1999) Genetic structure of Rhynchosporium secalis in Australia. Phytopathology 89:639645.

McDonald MC, Oliver RP, Friesen TL, Brunner PC, McDonald BA (2013) Global diversity and distribution of three necrotrophic effectors in Phaeosphaeria spp. New Phytologist 199:241-251.

Michelmore RW, Meyers BC (1998) Clusters of resistance genes in plants evolve by divergent selection and a birth-and-death process. Genome Research 8:1113-1130.

Read AF (1994) The evolution of virulence. Trends in Microbiology 2:73-76.

Sommerhalder RJ, McDonald BA, Mascher F, Zhan J (2011)
Effect of hosts on competition among clones and evidence of differential selection between pathogenic and saprophytic phases in experimental populations of the wheat pathogen Phaeosphaeria nodorum. BMC Evoutionary Biology 11:188, doi: 10.1186/14712148-11-188.

Stukenbrock EH, McDonald BA (2008) The origins of plant pathogens in agro-ecosystems. Annual Review of Phytopathology 46:75-100.

Van Baalen M, Sabelis MW (1995) The dynamics of multiple infection and the evolution of virulence. American Naturalist 146:881-910.

Wulff BBH, Horvath DM, Ward ER (2011) Improving immunity in crops: new tactics in an old game. Current Opinion in Plant Biology 14:468-76.

Zhan J, Mundt CC, Hoffer MH, McDonald BA (2002) Local adaptation and effect of host genotype on the evolution of pathogen: an experimental test in a plant pathosystem. Journal of Evolutionary Biology 15:634-47.

Zhan J, McDonald BA (2013a) Experimental measures of pathogen competition and relative fitness. Annual Review of Phytopathology 51:131-153.

Zhan J, McDonald BA(2013b) Field-based experimental evolution of three cereal pathogens using a mark-release-recapture strategy. Plant Pathology 62(Supp. 1):106-114. 